formed perhaps many thousands of years ago still exists and lies buried beneath subsequent annual layers, each of which is in some way typical of the age in which it was produced. Each layer will, for example, have sampled the atmospheric fallout appropriate to the time of its formation. Thus if the ice can be dated-as it often can by firn stratigraphy, oxygen isotope analysis or lead geochronology, for example-and its contents analysed, it is often possible to determine the pollution content of the atmosphere over periods of time.

This technique is particularly useful in determining the effects of man's activities upon the content of the atmosphere. It is well known, for example, that the burning of fossil fuels-oil, coal, lignite and natural gas-results in the transfer of various chemical elements and compounds from the continents to the atmosphere where they circulate and from which they ultimately fall. The analysis of dated ice samples thus means that today's atmospheric pollution can be compared with that of the recent past or even with the "standard" atmosphere before the industrial revolution. As a result, it is now known that the quantity of sulphur entering the atmosphere as a consequence of fossil fuel combustion is about the same as that arising from natural causes such as volcanic activity and the oxidation of organic matter.

Although sulphur may be the most severe of the polluting chemicals, what of other lesser elements? Selenium, for example, is emitted during the burning of fossil fuels and is, moreover, a member of the same group of elements as sulphur in the periodic table. So does it behave in a similar way to sulphur once it enters the atmosphere? To answer this question, Weiss et al. (Science, 172, 261; 1971) have analysed ice samples of fourteen different ages ranging from $800 \mathrm{BC}$ to the summer of 1965 for both sulphur and seleniumand have found that the proportions of each element which finally fall upon the ice sheets of Greenland and Antarctica are different. In pre-1960 ice samples, for example, the $\mathrm{Se} / \mathrm{S}$ ratios vary between 2.9 and $5.9 \times 10^{-4}$, whereas in post-1960 samples the corresponding range is 1.1 to $1.6 \times 10^{-4}$. The 1960 sample itself seems to be transitional with an $\mathrm{Se} / \mathrm{S}$ ratio of $2.2 \times 10^{-4}$. Thus although the combustion of fossil fuels has significantly increased the sulphur in the atmosphere in recent years, the selenium from the same fuels has not entered the atmospheric circulation to anything like the same extent. This assumes, of course, that much of the sulphur and selenium in the ice really has originated from the solid Earth, though this seems to be a warranted assumption. According to one study, $\mathrm{Se} / \mathrm{S}$ ratios in petroleum range between
0.31 and $1.22 \times 10^{-4}$ and the average ratio for coal is $2.9 \times 10^{-4}$. Moreover, most crustal rocks have ratios of the order of $10^{-4}$. In sea water, on the other hand, this ratio is usually three orders of magnitude smaller.

The conclusion to be drawn from all this is that the selenium from the burning of fossil fuel has not yet travelled over large distances, a situation which, when compared to the known behaviour of sulphur, is tantamount to saying that it hardly circulates about the atmosphere at all. Why should this be so? Weiss and his colleagues put it down to the chemical behaviour of the relevant tetravalent oxides. Sulphur forms the relatively stable gas $\mathrm{SO}_{2}$ upon oxidation whereas selenium forms $\mathrm{SeO}_{2}$, a white solid which is readily converted to red elemental Se at the sight of anything remotely resembling a reducing agent. On the other hand, biological processes at low temperatures result in volatile compounds of both sulphur and selenium. Selenium, for example, is volatilized from land plants as organic compounds of, as yet, unknown composition. Nevertheless, the proportion of $\mathrm{Se}$ in these forms is much smaller than that from fuels; and so most of it never reaches the colder regions of the Earth.

\section{ENVIRONMENT}

\section{Remote Sensors uf Work}

from a Correspondent

AT the seventh international symposium on remote sensing of environment held at the University of Michigan from May 17 to 21, the topics for discussion included instrumentation, data handling, automated photo interpretation and the application of remote sensing to geology, soil science, ecology, oceanography, meteorology, land planning and pollution monitoring.

Inevitably, considerable attention was directed to the Earth Resource Technology Satellite Programme (ERTS (A)) which is to be launched in March 1972 and several speakers outlined their own country's proposed participation in this programme. American workers re-

\title{
Transcriptional Control in Transformed Cells
}

THE precise state of the genome of the tumour virus in the transformed cell is still a mystery. In the cells that have been examined there are a few copies of the viral genome per cell (Gelb et al., J. Mol. Biol., 57, 129 ; 1971), which are probably integrated into the host's chromosomes, and Lindberg and Darnell (Proc. US Nat. Acad. Sci., 65, 1089 ; 1970) have shown that, in some cases at least, the SV40-specific mRNA molecules are considerably larger than those expected of a single-polycistronic transcript of the viral genome.

At the Lepetit Symposium in Paris last November, Darnell reported that these large mRNA molecules contained host-specific sequences as well as virusspecific sequences. This seemed to confirm the chromosomal location of the viral genome and suggested that the control of transcription of the integrated viral genome might differ from that of the autonomously replicating SV40 during a lytic infection.

This suspicion will now be strengthened further by the report of Sauer in next Wednesday's Nature New Biology. $\mathrm{He}$ has examined the SV40-specific mRNAs synthesized, in the presence of cytosine arabinoside, in an SV40-transformed monkey cell and a lytically infected monkey cell. Cytosine arabinoside is known to inhibit DNA synthesis and subsequent viral functions in the lytically infected cell and to restrict the synthesis of some mRNA molecules: the species of mRNA made in the presence of the inhibitor being defined as early mRNA. Sauer has found that in SV40 transformed cells treated with inhibitor, not only is early mRNA made but some late SV40 mRNA is also synthesized, in amounts which correspond to the transcription of about 10 per cent of the SV40 genome.

This surprising observation suggests that the physical state of the integrated viral genome has altered the nature of the sites at which viral RNA is initiated. This might occur by the simple act of removing the superhelical turns (if they exist inside the cell) or perhaps by fusing an otherwise untranscribed viral gene into a cell gene which is transcribed in the presence of cytosine arabinoside.

Sauer also observed that during a period of 18 months, the proportion of the virus-specific mRNA sequences which are transcribed in the SV40 transformed cell and which are homologous to SV40 late mRNA fell by about a half. Before the implications of these interesting observations are fully understood, much more information is needed about the nature of the integration of the tumour viral genome and the dependence of the initiation of transcription on the physical structure of the DNA template. 